\section{Endogenous retroviruses in amyotrophic lateral sclerosis}

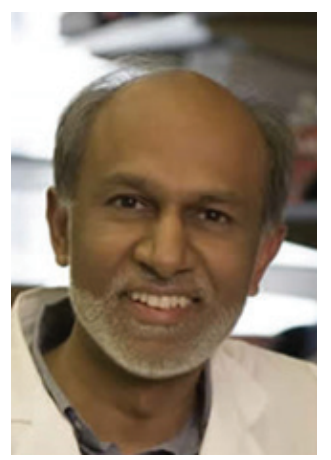

Avindra Nath* speaks to Elena Conroy, Commissioning Editor: Avindra Nath received his MD degree from Christian Medical College in India in 1981 and completed a residency in neurology from University of Texas Health Science Center in Houston (TX, USA), followed by a fellowship in multiple sclerosis and neurovirology at the same institution and then a fellowship in neuro-AIDS at National Institute of Neurological Disorders and Stroke. He held faculty positions at the University of Manitoba (1990-1997) (Canada) and the University of Kentucky (1997-2002) (USA). In 2002, he joined Johns Hopkins University (MD, USA) as Professor of Neurology and Director of the Division of Neuroimmunology and Neurological Infections. He joined NIH in 2011 as the Clinical Director of National Institute of Neurological Disorders and Stroke, the Director of the Translational Neuroscience Center and Chief of the Section of Infections of the Nervous System. His research focuses on understanding the pathophysiology of retroviral infections of the nervous system and the development of new diagnostic and therapeutic approaches for these diseases.

First draft submitted: 15 October 2015; Accepted for publication: 7 March 2016; Published online: 15 April 2016

\section{Q Could you give us a brief overview of your career to date?}

My major interests within neurology lie in infections and immune disorders of the nervous system. Following my medical training in India, I undertook a neurology residency, followed by a fellowship in multiple sclerosis (MS) in Houston (TX, USA) in the 1980s. In 1988, I joined the NIH in Bethesda (MD, USA) as a clinical fellow working on HIV infection of the nervous system, following which, in 1990, I became a faculty member at the University of Manitoba in Canada, continuing my research in HIV and MS. Subsequently, I spent 5 years (1997-2002) as a faculty member at the University of Kentucky (USA). In 2002, I became the director of the new Division of Neuroimmunology and Neurological Infections at John Hopkins University (MD, USA). I was also professor in the departments of neurology and neuroscience - positions I held for 9 years. Five years ago, I returned to the NIH as the clinical director of the National Institute of Neurological Disorders and Stroke where I am pursuing my work related to HIV infection and following my interests in endogenous retroviruses and undiagnozed neuroimmune disorders.

\section{Q What first drew you to the field of neurovirology?}

I began my residency in Houston (TX, USA) in 1983 around the start of the AIDS epidemic. During this time, I observed neurological complications exhibited by patients with AIDS, a phenomenon that had not previously been reported. I published these cases

*National Institute of Neurological Disorders and Stroke, National Institutes of Health, Bldg 10/ 7C-103, 10 Center Drive, Bethesda, MD 20892, USA; Tel.: +1 301496 1561; natha@ninds.nih.gov

\section{KEYWORDS}

- antiviral drugs • endogenous retroviruses $\bullet$ HIV/AIDS $\bullet$ mixed infections 
at about the same time as the viral basis of AIDS was discovered. So I became very interested to understand how and why a virus could cause such profound neurological manifestations. This subsequently drew me to the field of neurovirology. At that time, there were no laboratories where I could have carried out research into the relationship between HIV and the nervous system, so my first fellowship focused on the rubella virus pathophysiology while my clinical interest lay in MS. Rubella virus also causes numerous neurological complications and studying the virus provided me with useful background knowledge and a fundamental understanding in neurovirology and immunology. I then received the opportunity to become the first neuro-AIDS fellow at the NIH.

\section{Q What would you describe as the biggest achievement in your career?}

I feel like that is yet to come. Endogenous retroviruses are a relatively new area of investigation in my career and the moment I do not know where that will lead.

Looking at it retrospectively, the achievements we made while studying HIV pathogenesis have made a huge impact as we were one of the first to demonstrate that viral proteins can be toxic to neurons. We identified that the HIV Tat protein can be produced by cells despite antiretroviral therapy, initiating a cascade of events leading to neurocognitive deficits. We defined this work over 20 years by combining both bench and clinical research, and studied the mechanisms by which these proteins are produced and lead to neuroglial dysfunction, and have identified compounds that can be used to block these pathways.

I think the impact we had in trying to find reservoirs for HIV in the brain, as well as dissecting the mechanisms of neurodegeneration by HIV, are clearly major achievements that have resulted from work done in my laboratory.

Q One of your areas of interest is in controlling HIV reservoirs in the brain. Why is it that such little progress has been made in this area in terms of retroviral therapies?

For the US FDA to approve drugs you have to demonstrate that you can decrease viral load in the blood. It is not necessary to show that the drugs can impact the virus in the brain. Pharmaceutical companies have no incentive to look at the CNS as they are driven by profit margins. The reality is that you can control the virus in the periphery and the neurocognitive aspects will improve to some degree; however, neurocognitive deficits will continue to progress over longer periods of time. The challenge is that some of these drugs do not penetrate the CNS efficiently and thus viral replication and evolution may continue in the brain. Furthermore, even if one may be able control new virus from being formed, what cannot be controlled is the formation of some viral proteins, such as Tat. Although some of these proteins do not assemble all the way to form a virus, they can be released from the cells and be very neuro toxic. In the periphery, it might not be very critical but in the brain, where the neurons are most vulnerable, it is. Thus, existing antivirals are currently not sufficient to control the neurocognitive manifestations, so a different approach to controlling the production of the Tat protein is essential. Targeting this could have a huge impact on the neuropathogenesis of HIV infection.

Q You recently published a study implicating the HERV-K in amyotrophic lateral sclerosis; can you tell us a bit about this?

My clinical interest has always been in neurological infections and neuroimmune disorders. I often saw HIV patients who had unusual neurological manifestations. A few years ago, while I was at Johns Hopkins University, I came across a rare case in which a patient had both HIV and amyotrophic lateral sclerosis (ALS).

There were some interesting characteristics about this patient: first of all, he was very young (in his 20s) and ALS does not usually occur in that age group. He had contracted HIV in early childhood so in some ways he was a longterm survivor who suddenly had new, rapidly progressing, neurological symptoms. Within a month he lost a lot of motor function so the prognosis seemed poor and I was concerned that he was not going to die from the longstanding HIV infection but from ALS. Surprisingly, when we treated the patient for HIV, the ALS symptoms improved. When I searched the literature, I found similar cases of HIV-infected patients in whom ALS symptoms had improved with antiretroviral drugs. Prior research had also reported finding reverse transcriptase activity in sporadic ALS, even though the sample size of the patient population studied was small. In these reports they had also looked for exogenous retroviruses as a source of the reverse transcriptase but 
could not find any. This led me to reason that if the exogenous retroviruses could not be found, the reverse transcriptase might be arising from activation of endogenous retroviruses in the human genome. At that time, Jeffrey Rothstein, an expert in ALS, worked next to our laboratory and provided us with ALS brains to examine. We looked for a large panel of endogenous retroviruses by reverse transcriptase-PCR using published primers and we found that HERV-K RNA was consistently detected in the brain of ALS patients.

In 2011, we published a paper that demonstrated the association between HERV-K production in the brain and ALS. We did not find this in Parkinson's patients or in any individuals that had died suddenly from unrelated causes. These findings showed association between HERV-K and ALS, but did not prove causation: we did not know whether the death of neurons through neurodegeneration was activating the virus, or whether the virus was just a bystander. To answer these questions, we embarked on a larger study: we first tried to see whether we could synthesize a plasmid that contained the entire virus. We came up with a consensus sequence, made the virus and infected neurons in culture, which resulted in neurons dying. We reasoned that this was due to forcing the expression of a viral protein in the neuron. We then proceeded to try stimulating the endogenous retrovirus to find out whether that had an effect on the neurons. We used a modified CRISPRCas9 system and stimulated the production of the endogenous HERV-K, which also resulted in neuronal death. From our HIV studies, we knew that sometimes viral proteins were sufficient to cause toxicity. We worked on different viral proteins and found that the envelope region of the virus was sufficient to cause neuron death at the same rate as the full length virus - this made it much simpler to create transgenic animal models. We started by injecting brains of the animals in utero and observed that the virus was expressed in the neurons through a dysmorphic phenotype at birth. In addition, we carried out immunostaining on the brains of ALS patients and found that the HERV-K envelope was expressed in neurons but not glial cells. Therefore, we decided to express the envelope in the neurons of the animals via a neuronal promoter called Thy-1. We inserted the envelope gene using this promoter and created transgenic mice, which expressed the HERV-K envelope protein in all the neurons. However, the motor neurons degenerated, which was surprising as these animals had developed ALS affecting both upper and lower motor neurons. We found that the animals were born without symptoms and started developing the motor symptoms at approximately 3 months of age, which progressed gradually and eventually leading to a neurological death. This model expressed the phenotype of ALS much better than any other ALS mouse model that exists at the present time. We studied these mice very extensively: we did MRI scans, histopathology, western blots and all kinds of PCR analyses for transcripts to study the mechanism that was causing degeneration. We identified nucleolar dysfunction as being a critical component of this process.

\section{Q How does this retrovirus affect motor neurons in order to spread?}

I can only hypothesize about this as we do not have any experimental data as of yet. The hypothesis is as follows: the entire provirus can be expressed, so a viral particle could potentially be formed. If a viral particle is formed, there is the possibility that it can be released from a neuron and infect the adjacent neurons, which is how ALS actually spreads. If you observe ALS symptoms they seem to spread from neuron to neuron in the same anatomical region of the symptoms, which would be consistent with the hypothesis that the virus is being transmitted from one neuron to another via cell-cell contact. This indirect evidence exists, but it is yet to be proven.

Q Particularly exciting was the notion that a targeted therapy could be produced to treat ALS patients: how so?

There are multiple ways that this could happen: one is that we demonstrate that the envelope is neurotoxic and, therefore, it could be targeted through antisense molecules, via RNAi or antibodies. Alternatively, if this really is a complete retrovirus being transmitted, we should be able to develop treatments that are specific for the viral life cycle, as was done for HIV. There may be the possibility that some of the existing HIV drugs themselves may have some potential here but their effectiveness will have to be determined. We are currently carrying out a small clinical trial to investigate whether ALS patients treated with a combination of four antiretroviral drugs will experience a decrease in HERV-K 
levels. From our experience with HIV, we know that impacting the viral load a little is not effective. We do not expect our concoctions of antiHIV drugs are going to cure ALS, but if the viral loads drop by, say, just $50 \%$, it will give us impetus to modify and improve their efficiency.

\section{Q What are the next steps for this line of research?}

We are very interested in finding out why the virus gets activated in these patients. Are there any polymorphisms in the viral gene among ALS patients versus controls? How many copies of the virus exist? We are also looking to better define the mechanisms by which the virus causes toxicity.

\section{Q Are there any other viral causes of ALS?}

Numerous hypotheses have been suggested but none have come to fruition. A lot of viruses have been implicated in various neurological diseases but after some years these hypotheses tend to be forgotten. There are enteroviruses and polio-like viruses that have been implicated in ALS for a long time because ALS resembles polio in some ways, especially when it attacks the lower-motor neurons. Unfortunately, none of these efforts have proven a viral cause, but it is certainly not from a lack of trying.

\section{Q How do you see your field progressing in the next 5-10 years?}

It will be particularly exciting for the community to explore endogenous viruses in the context of various neurological diseases. In total, $8 \%$ of our human genome is composed of endogenous retroviruses but very few people currently study them. Most scientists that study them are geneticists, and they are interested in this from a genetic and an evolutionary stand point, but they are not studying it in the context of biological diseases other than cancer. I am quite hopeful that many researchers will start looking at that part of the genome in context of other pathological diseases much more closely - that alone would be a huge development over the next few years. Additionally, I foresee that a number of pharmaceutical companies will develop an interest in these viruses because they have been sitting on a huge array of drugs that were developed when they were screening for HIV drugs. They are very well positioned to pull those drugs off the shelf and start testing them against a whole host of these endogenous retroviruses, accelerating the drug development for these viruses very rapidly.

I believe we should also focus on rare diseases research over the next 5-10 years. I am hopeful that rare disease research will garner the appreciation and funding that it deserves. I have built a career through observing rare cases and going on to study them in the laboratory. In fact, many of the major advances against common diseases have occurred through the study of rare diseases. It is for this reason that I believe disease-focused funding is a mistake; we should fund the best science rather than the most common disease.

\section{Disclaimer}

The opinions expressed in this interview are those of the interviewee and do not necessarily reflect the views of Future Medicine Ltd.

Financial \& competing interests disclosure A Nath is an employee of the NIH and the US government. The author has no other relevant affiliations or financial involvement with any organization or entity with a financial interest in or financial conflict with the subject matter or materials discussed in the manuscript apart from those disclosed.

No writing assistance was utilized in the production of this manuscript. 